\title{
Sur les traces d'une petite croqueuse
}

\author{
Michèle Bolli
}

\begin{abstract}
A fictional tale - and/or a mirror enlarging a certain reality - such is the episode of a small woman's life who resists the misogyny-force that destroys her environment; misogyny that transforms beings into monsters, in this case into ogres. This text aims to question the form of resistances that can be opposed to these behaviors, inside as well as outside the feminine group. And, in either case, to begin by naming them, representing them, in one word making them visible to all, in order to transform them.
\end{abstract}

Il était un territoire peuplé d'êtres étranges qui avalaient tout sur leur passage. Là, chaque présence, chaque objet visible, était en danger de disparaître, absorbé.

Il arriva qu'elle entra en cet espace.

Elle observa la scène. Y vit d'abord les ogres-miroirs: des non-personnes qui rendent toute rencontre, tout échange de vues, impossibles. N'offrant à autrui, que miroitantes surfaces...

Puis, les monstres-bétonneurs: prenant l'autre sous une chape de béton, euxmêmes statufiés en hommes de pierre, réfractaires à toute atteinte sensible.

Pourtant, en ces lieux, il y avait aussi une maison-langage. Où se tenir à l'abri un moment. Le temps de prendre la mesure de ses forces. De réparer les avaries. De bâtir de nouvelles défenses peut-être, avant de repartir.

Restait un danger, ici, être mise à la porte, c'est-à-dire être réduite, parquée, classée, avant d'avoir repris suffisamment de force pour survivre au-dehors.

Toujours menacée donc, soit d'être absorbée, perdue dans l'un ou l'autre ventre d'ogre, soit d'être placée sous clôture... Menacée de parenthèse.

Un jour, sans bruit, elle partit.

Un ogre s'aperçut soudain que quelque chose lui échappait... Une petite partie manquait à l'ensemble. Son appétit n'était pas entièrement satisfait, ni sa volonté de dominer le territoire.

Irrité, il se mit à chercher. À chercher partout. De plus en plus fébrile. De plus en plus frustré. Il cherche, questionne, interroge, remue... Rien.

Pendant ce temps, une petite femme emporte avec elle la parcelle manquante. À belles dents, elle a croqué dans le petit $L u$ de la connaissance. Puis, s'est enfuie par une porte dérobée.

La voici, enfin délivrée de cet univers prédateur...

Commence sa vraie vie! corps.

À chaque étape, c'est un peu de son être propre qui émerge, qui prend 
$\grave{A}$ chaque pause, elles sont là, les voix mêlées: rient les bonnes fées, ricanent les carabosses (clin d'oeil à Pinget, mais aussi réalité).

Plus loin, elle croisa une vieille qui lui prédit une pluie de catastrophes si elle persistait à déployer son propre mouvement, à vivre en liberté.

Mais la petite croqueuse fit la sourde oreille et construisit sa première cabane.

Elle avança plus encore...

Surgit une Carabosse médusante. Elle la fixa et chercha à l'immobiliser par son regard glaçant.

Évitant de croiser ses yeux, la petite croqueuse s'échappa pour construire sa seconde cabane.

Après un long parcours, elle croisa une carabosse curieuse-insatiable... Celle-ci questionnait ses victimes jusqu'à leur pâmoison.

Résistant une fois encore, la petite croqueuse lui tourna le dos, avança rapidement jusqu'au lieu où elle construisit sa troisième cabane.

Enfin, un visage aimé se rapproche. Longtemps rêvé. N'est-il une fois encore qu'un leurre?

Lestée de sa croque, la jeune femme s'avance vers lui, tout à la fois contente de son acquis, et craintive à l'égard des événements à venir. Il s'avance en souriant: «Ogre ou compagnon-bâtisseur?» se demandait-elle.

Mais de l'horizon, à grandes foulées, déjà s'approche la Carmen... Carabosse encore, mais jeune, impétueuse, impérieusement désirante...

Elle écrasa la petite croqueuse...

Ratatinée au sol, mais pas morte, la petite croqueuse rampa hors de la vue de la rouge Carmen.

Se redressa. Se défroissa. Secoua ses boucles. Sortit son rouge à lèvres à petit miroir serti de grenats. Redessina la forme fruitée de sa bouche. Puis, reprit sa route en imaginant la forme de sa prochaine cabane.

(extrait d'un livre à paraître) 